\title{
ERRATUM
}

\section{Erratum to: A Glycine soja ABA-responsive receptor-like cytoplasmic kinase, GsRLCK, positively controls plant tolerance to salt and drought stresses}

\author{
XiaoLi Sun • Mingzhe Sun • Xiao Luo • \\ XiaoDong Ding $\cdot$ Hua Cai $\cdot$ Xi Bai • \\ XiaoFei Liu $\cdot$ YanMing Zhu
}

Published online: 3 September 2014

(c) Springer-Verlag Berlin Heidelberg 2014

Erratum to: Planta (2013) 237:1527-1545

DOI 10.1007/s00425-013-1864-6

The co-author Wei Ji withdraws his authorship, therefore the correct author group is listed below.

XiaoLi Sun · Mingzhe Sun - Xiao Luo - XiaoDong Ding · Hua Cai $\cdot$ Xi Bai $\cdot$ XiaoFei Liu $\cdot$ YanMing Zhu

Regrettably, incorrect versions of Figs. 6a and 7a are used in the original publication. The correct versions of Figs. 6a and $7 \mathrm{a}$ are given below.

The online version of the original article can be found under doi:10.1007/s00425-013-1864-6.

X. Sun $\cdot$ M. Sun $\cdot$ X. Luo $\cdot$ X. Ding $\cdot$ H. Cai $\cdot$ X. Bai $\cdot$ X. Liu $\cdot$

Y. Zhu ( $\varangle)$

Plant Bioengineering Laboratory, Northeast Agricultural

University, Harbin 150030, China

e-mail:ymzhu@neau.edu.cn; ymzhu2001@neau.edu.cn

X. Ding

Department of Neurology, The University of Texas

Southwestern Medical Center, Dallas, TX 75390, USA 
Fig. 6a

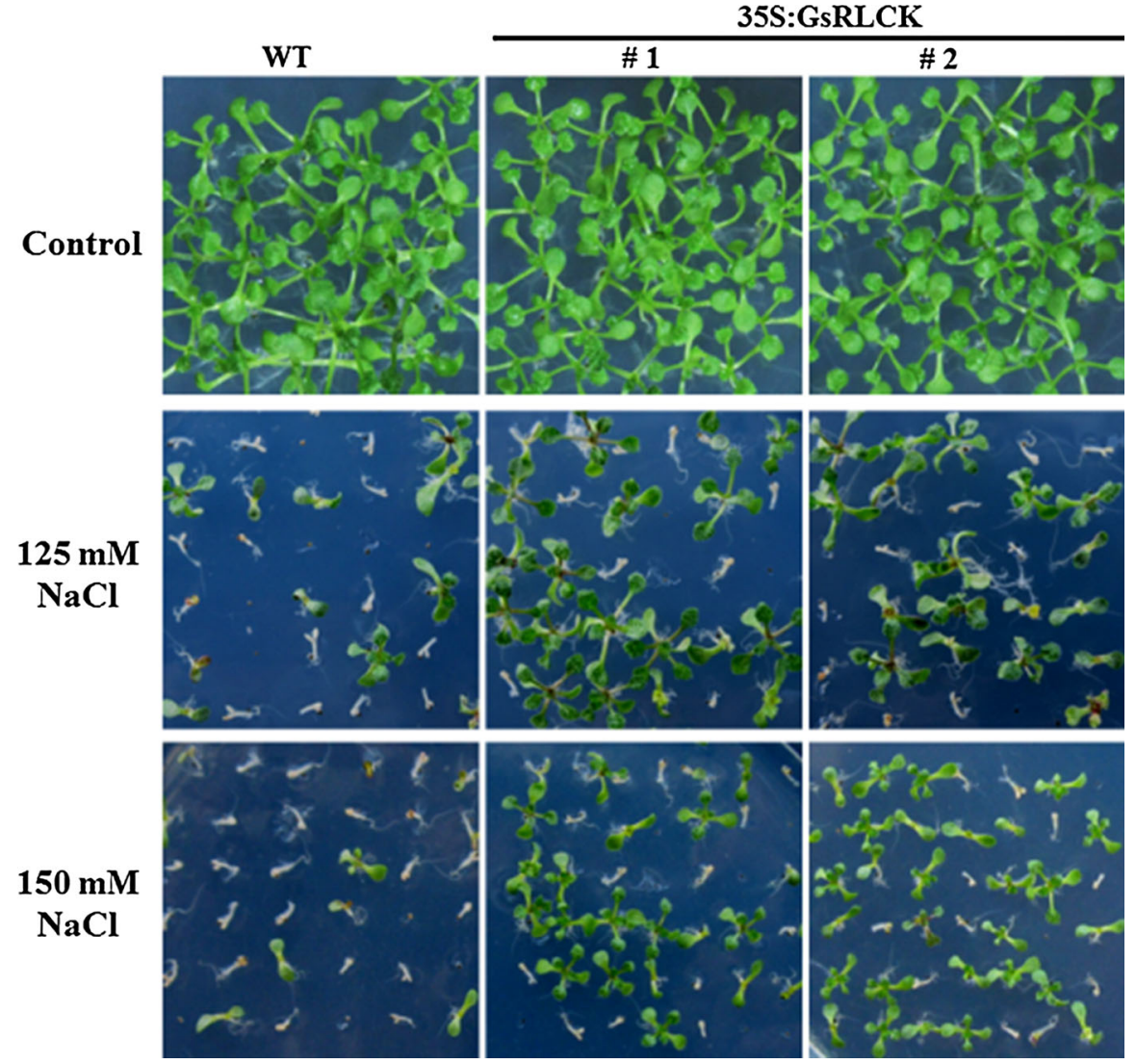

Fig. 7a

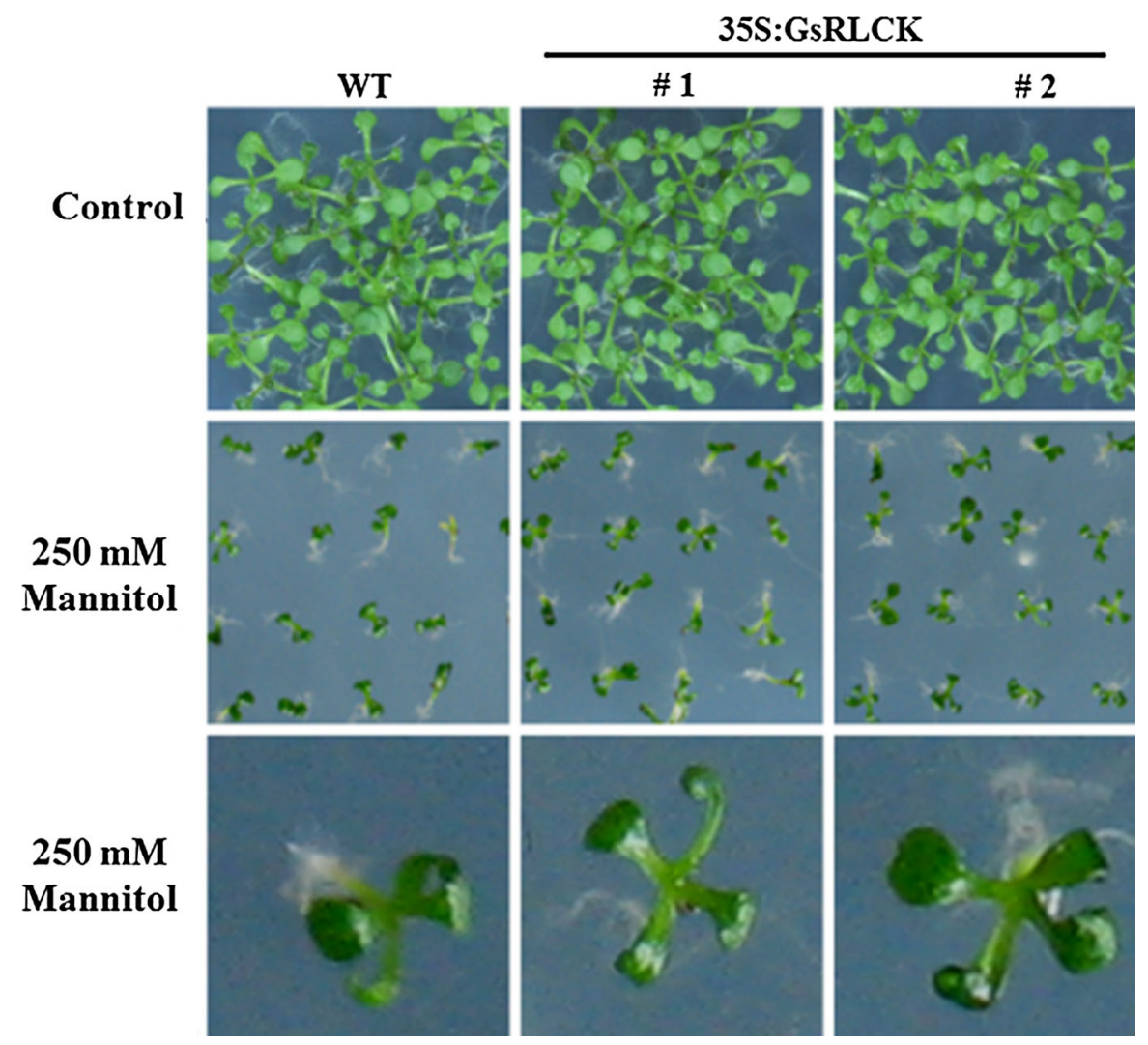

